\title{
Educación afectivo-sexual en las escuelas familiares: un asunto sobre la construcción vital ${ }^{1}$ Affective-sexual education in family schools: A matter about vital construction
}

Alexander Rodríguez Bustamante*, Joaquín Parra Martínez ${ }^{* *}$, María Ángeles Gomariz Vicente ${ }^{* * *}$, Johanna Jazmín Zapata Posada ${ }^{* * * *}$, Zulema Elisa Rodríguez Triana ${ }^{* * * *}$, Juan Carlos Echeverri Álvarez

\section{Forma de citar este artículo en APA:}

Rodríguez Bustamante, A., Parra Martínez, J., Gomariz Vicente, M. A., Zapata Posada, J. J., Rodríguez Triana, Z. E., \& Echeverri Álvarez, J. C. (2021). Educación afectivo-sexual en las escuelas familiares: un asunto sobre la construcción vital. Poiésis, (41), 24-42. https://doi.org/10.21501/16920945.4185

\section{Resumen}

El texto surge a través de las reflexiones conceptuales en el Doctorado en Educación de la Universidad Pontificia Bolivariana, en Medellín, Colombia, y la Pasantía Doctoral en la Universidad de Murcia con el grupo de investigación "Compartimos Educación" con el objetivo de caracterizar el lugar de las escuelas familiares en la promoción de la educación afectivo-sexual de los niños, niñas y adolescentes en la escuela. La metodología

Pasantía Internacional en cooperación interinstitucional entre la Universidad Pontificia Bolivariana y su Doctorado en Educación (Medellín, Colombia) y la Universidad de Murcia con el grupo de Investigación "Compartimos Educación" del Departamento de Métodos de Investigación y Diagnóstico en Educación; Facultad de Educación. Este artículo a su vez se deriva de la investigación doctoral en curso titulada "Familia y escuela: educación afectivo sexual en las Escuelas de Familia", del estudiante Alexander Rodríguez Bustamante adscrita al Doctorado en Educación de la Universidad Pontificia Bolivariana (Medellín-Colombia). Texto leído en la la XLI Lectura de ensayos de la Facultad de Psicología y Ciencias Sociales de la Universidad Católica Luis Amigó (Medellín-Colombia). Experiencia de Pasantía Internacional en la Universidad de Murcia-España

Doctorando en Educación de la Universidad Pontificia Bolivariana (Medellín-Colombia). Estudiante de Pasantía en el grupo de investigación "Compartimos Educación" de la Universidad de Murcia (España) del Departamento de Métodos de Investigación y Diagnóstico en Educación. Director de la Escuela de Posgrados de la Universidad Católica Luis Amigó (Medellín-Colombia). Miembro del grupo de investigación "Farmacodependencia y otras adicciones" de la Facultad de Psicologia y Ciencias Sociales de la misma Universidad. Contacto: alexander.rodriguez@upb.edu.co, https://orcid.org/0000-0001-6478-1414

Doctorando en Educación de la Universidad Pontificia Bolivariana (Medellín-Colombia). Estudiante de Pasantía en el grupo de investigación "Compartimos Educación" de la Universidad de Murcia (España) del Departamento de Métodos de Investigación y Diagnóstico en Educación. Director de la Escuela de Posgrados de la Universidad Católica Luis Amigó (Medellín-Colombia). Miembro del grupo de investigación "Farmacodependencia y otras adicciones" de la Facultad de Psicología y Ciencias Sociales de la misma Universidad. Contacto: alexander.rodriguez@upb.edu.co https://orcid.org/0000-0001-6478-1414

Ph.D Philosophy and Education Sciences. Departamento de Métodos de Investigación y Diagnóstico en Educación; Facultad de EducaciónUniversidad de Murcia (España). Miembro del Grupo Investigación "Compartimos Educación" Contacto: jparra@um.es hittps://orcid. org/0000-0001-6818-8909

PhD en Pedagogía. Departamento de Métodos de Investigación y Diagnóstico en Educación; Facultad de Educación-Universidad de Murcia (España). Miembro del Grupo Investigación "Compartimos Educación" Contacto: magovi@um.es https://orcid.org/0000-0002. 8308-0848

PhD en Desarrollo y Ciudadanía: Derechos Humanos, Igualdad, Educación e Intervención Social - Universidad Pablo De Olavide. Investigadora de la Universidad Pontificia Bolivariana (Medellín-Colombia). Miembro del grupo de investigación en Familia de la Facultac de Trabajo Social de la UPB. Contacto: johanna.zapata@upb.edu.co https://orcid.org/0000-0003-3897-0451

PhD en Ciencias de la Educación-Universidad de Caldas. Investioadora de la Universidad de Caldas (Manizales-Colombia). Departamento de Estudios de Familia, Facultad de Ciencias Jurídicas y Sociales, Manizales, Colombia. Contacto: zulema.rodriguez@ucaldas.edu.co https://orcid.org/0000-0003-2379-6278

PhD en Educación de la Universidad Pedagógica Nacional, Bogotá-Colombia. Docente Titular Universidad Pontificia Bolivariana (Medellín-Colombia). Director del Doctorado en Educación de la UPB. Director del Grupo Investigación Pedagogía y Didácticas de los Saberes (PDS). Contacto: juan.echeverri@upb.edu.co https://orcid.org/0000-0001-9577-468X 
es de corte cualitativo. Como resultado se muestra la necesidad de vincular actores que transversalizan el quehacer de los sujetos, la familia y escuela en un lugar común, las escuelas familiares para la promoción de la educación afectivo-sexual para gestar acciones de cambio individual y social. Es evidente la necesidad intrínseca de generar la discusión en cuanto al género para la deconstrucción de prácticas socioculturalmente atribuidas, promover espacios formativos respecto a la sexualidad y la afectividad e incentivar a la reflexión como ejercicio pedagógico.

\section{Palabras clave:}

Afectividad; Educación; Educación afectivo-sexual; Escuelas familiares; Pedagogía; Relación familia-escuela; Sexualidad.

\section{Abstract}

This text emerges through the conceptual reflections of the Doctorate in Education at Universidad Pontificia Bolivariana, in Medellín, Colombia, and the Doctoral Internship at University of Murcia with the research group "Compartimos Educación" with the aim of characterizing the place of family schools in the promotion of affective-sexual education of boys, girls and adolescents at school. The used methodology is qualitative. As a result, the need to link actors that mainstream the work of the subjects, family and school in a common place, the family schools for the promotion of affective-sexual education to create actions of individual and social change is shown. The intrinsic need to generate discussion regarding gender for the deconstruction of sociocultural attributed practices, promote training spaces regarding sexuality and affectivity and encourage reflection as a pedagogical exercise is evident.

\section{Keywords:}

Affective-sexual education; Affectivity; Education; Family-school relationship; Family schools; Pedagogy; Sexuality. 


\title{
Introducción
}

\begin{abstract}
Se plantean lugares de encuentro entre estos dos sistemas interconexionados por el pensamiento sistémico y la comunicación, rescatando que la escuela y la familia han sido determinantes en la configuración de subjetividades y sociedades. Estas reflexiones permiten concluir que las manifestaciones recíprocas de la familia y la escuela esculpen maneras de ser y de estar en el mundo. (Rodríguez et al., 2021, p.374).
\end{abstract}

El desarrollo humano y educativo de los sujetos tiene orígenes en dos ámbitos en los que se desenvuelven los sujetos a lo largo de su vida, la familia y la escuela; es claro pensar que en cada uno de los escenarios se adquieren competencias, valores, creencias y costumbres donde se fomentan capacidades y habilidades que sustentan su hacer en prácticas interaccionales para el devenir del ser en sociedad desde la libertad, autonomía y (auto) referencia.

El ejercicio realizado por los sujetos va acompañado de actores significativos que a través de técnicas, herramientas y metodologías, favorecen para el proceso. Son las familias y las escuelas los actores llamados a ocupar el lugar para el desarrollo y fortalecimiento desde lo educativo. Si bien, tradicionalmente este proceso se ha visto fragmentado y ha generado repercusiones en el actuar cotidiano de las personas, en la actualidad, se estudian múltiples formas para fortalecer la relación familia-escuela como un escenario que agencia la interacción humana.

Allí, emergen las escuelas familiares como estrategias de participación de las familias en la institucionalización en pro de los niños, niñas y adolescentes acompañados de sus maestros (Gomariz Vicente et al., 2019); en otras palabras, se convierte en el lugar idóneo para vincular los actores familiares e institucionales a un llamado de reflexión y actuación conjunto en situaciones diversas para el proceso de desarrollo humano y educativo de las personas.

Así las cosas, en las escuelas familiares se trabajan temas que están directamente relacionados con las necesidades de cada actor, gestor y promotor de desarrollo en materia de educación, sean maestros, grupos familiares o niños, niñas y adolescentes. En esta línea, los agentes educativos se enfrentan con una educación primaria impartida por la familia de origen y crianza, relacionada con el hecho de la enseñanza de valores, creencias y experiencias que generan nuevos aprendizajes colectivos, como elementos que movilizan acciones educativas en la institucionalidad a través de estrategias pedagógicas que generen cambios estructurales.

Específicamente para el espacio dialógico, los temas relacionados con la afectividad y la sexualidad que son los que llaman la atención en el texto, la familia ha iniciado un proceso educativo desde la historia, tradición y cultura, que le dan significado a estos dos conceptos (López, 2016), y la escuela trabaja estos temas desde una perspectiva histórica, narrativa, vocálica y socio cultural, 
propuestas anteriores descritas por Rodríguez Bustamante \& Viveros Chavarría (2013); en tal sentido surge la necesidad de revisar que tanto la familia como la escuela se conectan alrededor de una educación afectivo-sexual que no oprima, limite, desinforme o subyugue la libertad que tiene cada sujeto de respetar y ser respetado por el otro, es decir, de reconocer las diversidades en sus máximas expresiones. Para eso existen

aquellos/as que sí han tratado la educación sexual en casa, dicen haber hablado, principalmente acerca del uso de anticonceptivos, embarazo e infecciones de trasmisión sexual, es decir, temas muy relacionados con la salud. [...] hace falta una mayor concienciación acerca de las redes sociales y sus peligros. (López, 2016, p. 64)

Adicionalmente, es transversal la categoría de género como una construcción social e histórica (Lamas, 1996) que tiene que ver con el modelo educativo desde el cual se promueve la educación afectivo-sexual en las instituciones para motivar relaciones basadas en el respeto, reconocimiento y prevención de la discriminación y violencia en sus múltiples representaciones, propósito por el cual, tanto la familia como la escuela le apuestan a la educación para la (re)construcción de un tejido social.

En esta línea, Barrientos et al. (2018) generan una propuesta conceptual donde surge la coeducación como estrategia para trabajar las desigualdades que se presentan en la sociedad a partir de la escuela; lo cual se convierte en una manera en que los maestros y maestras puedan poner sobre la mesa el tema de la educación afectivo-sexual como elemento interaccional, humano y parte del conocimiento crítico y experiencial del mismo docente, es decir, hay una puesta en escena de sí mismo en el ejercicio práctico frente a las inequidades entre hombres y mujeres.

Con base en lo anterior, el presente artículo tiene la pretensión de mostrar un posible camino para desarrollar la educación afectivo-sexual a través de las escuelas familiares como lugar que conecta la vida de los niños, niñas y adolescentes con la familia y la escuela. El desarrollo se realiza en tres acápites articulados. Primero, se hace un acercamiento a lo que significaría la educación afectivo-sexual y su implicación conceptual donde se pueden generar estrategias pedagógicas que sirvan tanto para estudiantes como para los maestros en la resignificación de la afectividad y la sexualidad.

Segundo, se muestran las maneras en que las familias y las escuelas han participado en los procesos educativos; se muestran las fronteras desde las cuales han trabajado individualmente $y$ el punto que las conecta, es decir, se hace referencia a las escuelas familiares como lugar común de conversaciones humanas, educativas, reflexivas y agenciadoras de desarrollo humano y social.

Finalmente, se desarrolla la temática de las escuelas familiares como un territorio de construcción en educación afectivo-sexual, visibilizando los beneficios que tiene para los sujetos poner sobre la mesa temas considerados tabú para la institucionalidad que no se han trabajado con anterioridad en estos escenarios y la forma en que participan tanto los grupos familiares como las escuelas. 
A manera de conclusión, se enfatiza en la importancia del trabajo articulado que se debe promover en la relación familia-escuela y la forma en que la educación afectivo-sexual trae aciertos en el desarrollo del ser y estar en sociedad, abordada desde las escuelas familiares.

\section{La educación afectivo-sexual: una realidad y, una responsabilidad}

Existen estudios que se han aventurado a abordar la temática de la educación relacionada con aspectos de lo sexual y un poco se adentran al componente afectivo del ser humano (Lazo Corvera \& Collignon Goribar, 2017; Garín et al., 2016; Langa Herrero, 2019); lo que da significado al lugar que ocupa la educación en el proceso de vida de las personas; si bien, se comprende como un proceso de enseñanza y aprendizaje de un maestro o maestra a sus alumnos sobre algún tema en específico, esta actividad no termina allí, pues es mucho más compleja. El proceso mismo no se limita únicamente al intercambio de saberes, sino a las relaciones humanas que se tejen en la práctica educativa de las interacciones cotidianas; en este punto del escrito se propone que los maestros continúen en construcción con las familias y el alumnado a partir de un tema que tiene una directa vinculación subjetiva, la sexualidad y el afecto.

La Organización Mundial de la Salud (2006) recoge la educación afectivo-sexual como un derecho de niños, niñas y adolescentes, junto a otros derechos sexuales como son: la libertad, la privacidad, la equidad, la convivencia en igualdad y sin discriminaciones, postura que adopta en su análisis para-construcción de sociedad. En esta línea, se verá en el presente acápite como una apuesta, una propuesta y una oportunidad tanto para las familias, el profesorado como para el alumnado. La educación afectivo-sexual en este escrito se comprende de manera intercultural, relacional, humanista (Carmona Sáez et al., 2021) y hace referencia a toda aquella conexión subjetiva relacionada con el ser y hacer sociedad sobre la sexualidad y afectividad; manera en que cada persona genera reflexiones internas y colectivas para vincularse con otro lo cual le propicia un papel liberador y autónomo. En este sentido, pensar la educación desde el lente de las relaciones intersubjetivas significa sumergir la mirada a un campo de conocimiento complejo, inacabado y movedizo conceptualmente y valoralmente hablando.

Específicamente, el tema de la educación afectivo-sexual ha cogido mayor fuerza en la actualidad debida la implicación e importancia que tiene la temática misma para el desarrollo humano; la limitación en la que ha estado sumergida a lo largo del tiempo gira en torno a los miedos, prejuicios, estereotipos y repercusiones que tiene para el maestro enfrentarse a una realidad (Bejarano Franco \& García Fernández, 2016); la idea sería seguir la línea que propone Rosales Mendoza y Flores Soriano (2009) en mostrar la necesidad de que las instituciones de educación básica, media y superior apuesten a desarrollar la sexualidad de carácter formativo y no limitarse únicamente a lo informativo. Además, aportar a la construcción del ser en libertad es mediante la educación afectivo-sexual, puesto que es un elemento que le dará bases de relacionamiento humano, cálido y democrático a cada persona (González Gómez, 2015). El proceso que se debe realizar en las aulas 
debe partir de la reflexión y la autonomía para tejer conocimiento, pasando del bagaje sociocultural que han aprehendido las familias, NNA, maestros y maestras, y en este entramado, de experiencias en que se desenvuelve cada uno.

Dicho lo anterior, se realiza una propuesta para abordar la educación afectivo-sexual en las instituciones educativas acompañada del enfoque de derechos humanos; hablar de sexualidad y afecto en estos espacios es garantizar el derecho a la libertad de expresión, autonomía, libre albedrío, entre otros; y en esta línea,

una buena y, sobre todo, temprana educación afectivo-sexual en las etapas educativas puede ayudar a suprimir roles, estereotipos y actitudes de corte racista, homófoba y xenófoba con el fin de prevenir y evitar discriminaciones, malos comportamientos en relación al sexo y/o al género, etnia, cultura, religión o ideología. (Bejarano Franco \& Mateos Jiménez, 2015, p. 1508)

En este ejercicio es importante un proceso de reflexión, resignificación y libertad de los docentes, familias y alumnado, en cuanto se despojan de los prejuicios implicados en su propia vida y realidad. Realizado el anterior ejercicio, es posible que las escuelas permitan potenciar la educación afectivosexual como una herramienta significativa de democracia y humanidad (González Gómez, 2015). Aquí se ve la relevancia de incluir la educación afectivo-sexual en las aulas de clase para generar una sociedad libre de moralismos, para ir construyéndola entre los partícipes. Bajo los anteriores planteamientos, familia y escuela a través de una relación dialógica deben ser, como lo expresan Del Pozo et al. (2020), "escenarios de democratización en los que se aprenda a vivir en el encuentro y se niegue cualquier forma de violencia y discriminación" (p. 176), al tiempo que, para el este caso, reconozcan el género y la construcción de vínculos para la educación afectivo-sexual como parte constitutiva de los procesos que en común puedan implementar. Las escuelas familiares, en este sentido, se convierten en una mediación pedagógica para incluir un tema que pocas veces se contempla en esta estrategia y que, generalmente, se asume como un contenido de aprendizaje solo para los-as estudiantes y no se incluye a las familias, y en ellas, su relación con los- maestros y maestras.

Así, La versatilidad conceptual y metodológica que tiene la temática se basa en el abordaje diverso, dependiendo de su grupo poblacional, desde niños, niñas y adolescentes hasta grupos de adultos específicos. Allí, se pueden trabajar temáticas que van desde la intimidad, traspasa lo familiar y confluye en lo social, como pueden ser vistas en la siguiente tabla. 


\section{Tabla 1}

Relación población-tema en perspectiva de la educación afectivo sexual

\begin{tabular}{lll}
\multicolumn{1}{c}{ Población/Tema } & \multicolumn{1}{c}{ Afectividad } & \multicolumn{1}{c}{ Sexualidad } \\
\hline Maestros y maestras & $\begin{array}{l}\text { Relaciones humanas } \\
\text { Vínculos colaborativos desde la institucionalidad. }\end{array}$ & $\begin{array}{l}\text { Relación sexo/género } \\
\text { Formación en género y teorías feministas }\end{array}$ \\
\hline \multirow{2}{*}{ Grupos familiares } & $\begin{array}{l}\text { Vínculos afectivos en las familias } \\
\text { Bagaje histórico-cultural. }\end{array}$ & $\begin{array}{l}\text { Sistema de creencias } \\
\text { Estereotipos de género y sexualidad } \\
\text { Reconocimiento de la diversidad }\end{array}$ \\
\hline \multirow{2}{*}{ Niños, niñas y adolescentes } & $\begin{array}{l}\text { Reconocimiento del otro y vinculación afectiva } \\
\text { Amigos de confianza. }\end{array}$ & $\begin{array}{l}\text { Reconocimiento del cuerpo } \\
\text { Derechos fundamentales en sexualidad }\end{array}$ \\
\hline \multirow{2}{*}{ Escuelas familiares } & Construcción de proyectos de vida & $\begin{array}{l}\text { Deconstrucción de una relación basada en la } \\
\text { sexualidad } \\
\text { La hipersexualización infantil }\end{array}$ \\
\hline
\end{tabular}

Hasta aquí, se podría pensar la realización de una propuesta metodológica tentativa que cumpla el objetivo de generar escenarios de reflexión, resignificación y autonomía en lo que respecta a la sexualidad y la afectividad para familias maestros, maestras y alumnado. Por su parte Font (2010), comprende que la educación afectivo-sexual tiene que ver con la incorporación de nuevas instrucciones a modo de preparación para la vida adulta, y en el ámbito educativo que vincula a la familia, menciona que "está relacionada con una concepción holística de la sexualidad y de la educación sexual basada en un modelo que incluye la interacción entre los diferentes aspectos físicos, psicológicos y sociales" (p. 6).

En el caso del proceso de pasantía se proponen cinco caminos para el cumplimiento de lo planteado en este apartado en una línea de tiempo que constituye un antes, durante y después de un ejercicio educativo y pedagógico; nominado de la siguiente manera:

- La planificación sexo-afectiva,

- Preparación o encuadre,

- Desarrollo pedagógico colaborativo,

- Resignificación y reflexividad bidireccional,

- Sistematización de los aprendizajes,

Uno, la planificación sexo-afectiva. Se detectan estructura y característica, necesidades, dinámicas de los grupos poblacionales; tiene en cuenta su estructura y característica, necesidades, dinámicas y resultados esperados frente a la práctica que será desarrollada en el escenario. Aquí el maestro tiene la labor de convertir un tema que se ha trabajado históricamente en línea informativa, a uno formativo; debe revisar las formas en que se ha realizado el trabajo e indagar sobre nuevos lineamientos de acción, formas de abordaje, instrumentos y herramientas para generar un proceso consciente de reflexión y cambio.

Dos, la preparación o encuadre. Es donde las familias, el maestro o la maestra, conjuntamente, después de realizar un ejercicio previo libre de prejuicios frente al trabajo que se llevará a cabo, han pensado la mejor forma de desarrollarlo que dinamicen las personas en el escenario; proponen 
el diálogo, la comunicación bidireccional activa, las temáticas y los escenarios de discusión del aprendizaje colaborativo. Se convierte en un momento clave para conocer las necesidades de la población y revisar la forma para encarar el reto.

Tres, desarrollo pedagógico colaborativo. Aquí las familias, maestros y maestras se enfrentan a las realidades que los alumnos tengan con relación a la sexualidad y afectividad, puesto que se visibilizarán experiencias que se hayan adquirido a lo largo de la vida y que estén relacionadas con el bagaje histórico-cultural. Se apoyarán en herramientas técnicas que consideren necesarias para llevar a cabo los objetivos propuestos para la temática.

Cuatro, resignificación y reflexividad bidireccional. Este momento implica un durante en la relación educativa donde se tejen en conjunto aprendizajes que resignifiquen experiencias y concepciones tradicionalmente atribuidas, mediante una relación bidireccional temática, pensada e interiorizada; se gestionen escenarios reflexivos para reaprender aspectos relacionados a la sexualidad y la afectividad. Los NNA expresan sus temores y dudas en la deconstrucción de los aprendizajes; las familias están llamadas a la conversación cuando se convierte en uno de los ámbitos que más infiere en la construcción cognitiva y emocional de los sujetos.

Cinco. Sistematización de los aprendizajes. Aquí las familias, los maestros, maestras y el alumnado se enfrentan a un antes y después de un ejercicio educativo y pedagógico frente a temas relacionados con la sexualidad y la afectividad. Sirve como evaluación y potencia la resignificación en tanto muestra los cambios que se han generado a través del diálogo, la interacción y el aprendizaje colaborativo.

\section{Familia y escuela, agentes participantes en la educación afectivo-sexual. Una tensión que persiste y muta}

El subtítulo en particular, y el artículo en general, establecen una relación que en la actualidad parece natural y necesaria. Es decir, no sorprende a nadie, en espacios académicos educativos, invocar esta relación, hacer prescripciones, proponer innovaciones, elaborar propuestas y formular investigaciones. Sin embargo, en términos históricos, ni siquiera es fácil y transparente el vínculo entre la familia y la escuela; tampoco es natural que la escuela se encargue de la educación sexual: aspecto de la vida que durante mucho tiempo se les negó a los NNA; y mucho menos es evidente que, juntas la escuela y la familia, aúnen esfuerzos de relacionamiento para educar en un terreno como el de la sexualidad y los afectos como si estos elementos de la vida personal fueran otros de los saberes que ingresan a la escuela para ser enseñados con efectos de utilidad económica; nada tiene de natural hablar de escuelas familiares: escuela y familia han sido dos espacios diferentes que han compartido un objeto vinculante, pero, al mismo, generador de distancias problemáticas entre ambos: los niños o la infancia. 
Por supuesto, este no es el espacio para hacer una aproximación histórica a la relación familiaescuela, porque el objetivo es claramente otro: mostrar las potencias de esta relación en la vida individual de los estudiantes y, por tanto, mostrar que la formación afectivo sexual debe ser una empresa común de estas dos instituciones sociales. Empero, sí es importante señalar algunos puntos que permitan reconocer que se es inocente en cuanto a las suspicacias, las objeciones y los interrogantes que surjan en torno a las nuevas emergencias, las tareas emergentes y las demandas que brotan en las lógicas de esta vieja y problemática relación entre familia y escuela. Dicho de otra forma, no suponemos que la escuela, y ni siquiera la familia, hayan sido los espacios históricamente destinados a la formación afectivo-sexual, pero denunciamos que hasta ahora lo han hecho de muy mala manera 0, por lo menos, que por algún motivo habían descuidado esta función vital. Error histórico que se podría corregir en el nuevo territorio de las escuelas familiares que analizamos aquí.

La familia y la escuela han sido desde la transición del antiguo régimen a la formación de los estados constitucionales — vigentes todavía — dispositivos para el gobierno de la población. En primera instancia fueron los espacios para afrontar la cuestión social, el problema de los pobres, como una forma de producir y al mismo tiempo controlar la libertad necesaria para vivir la vida en las lógicas republicanas y democráticas. La familia misma no es el universal que pueda servir como núcleo intocado de diferentes formas de sociedad y de poder: ella es el resultado de diferentes discursos y prácticas, complejo juego de estrategias y de fuerzas que interactúan con base en múltiples puntos fuera y dentro de la misma familia. La escuela, por su parte, también hace parte del dispositivo para regular los comportamientos educativos de los hijos convertidos en escolares y reforzar su conversión en ciudadanos. Por tanto, la relación es presumible pero no fácil.

Ahora bien, parte de ese gobierno de la población, en la familia y en la escuela, pasa por coordenadas como la medicina, la higiene, la sexualidad. Esta sexualidad, inicialmente, era cuestión de la medicina y del sacerdote: para la familia era atavismo, para la escuela inexistencia 0 anormalidad. Para ambos, familia y escuela, el encargo general era propiciar la mejor adecuación de la población a las transformaciones de la sociedad en términos de desarrollo económico, científico y tecnológico. Familia y escuela, entonces, han sido dispositivos que hacen cada vez más exhaustivos sus mecanismos de control y de gobierno dentro de los estados constitucionales en relación con las realidades y tendencias a escala global. Esta exhaustividad estuvo marcada durante el siglo XX, y todavía en la actualidad, por la emergencia de disciplinas "psi". A mediados de ese siglo, a partir del psicoanálisis, el deseo fue convertido en un dominio legítimo de intervención en el cual se conjugaban los problemas de rendimiento escolar con los de una mejor gestión de la familia, es decir, intervenciones "psi" que articulan los ideales familiares con las exigencias sociales de gestión de la población.

Ahora bien, pese a que la escuela y la familia han sido dos instancias constitutivas y constituidas por las formas democráticas de gobierno, esto no significa que entre ellas haya existido 0 exista unas relaciones transparentes y armónicas en el norte de un objetivo común, por el contrario, aunque ese objetivo común existe, esto es, la cualificación constante de la democracia, sus caminos son diferentes, antagónicos en muchos casos e, inclusive, lo que parece una relación evidente, 
la mar de las veces es una no-relación que tiene que ser constantemente pensada, interrogada, intervenida, para que la sí-relación realmente tenga elementos comunes y no esté definida, simplemente, por el movimiento que se genera por antagonismos entre las dos instancias. Es una lucha que todavía se sostiene, pero que ha tenido diferentes expresiones en el tiempo. Durante el siglo $\mathrm{XIX}$, por ejemplo, el discurso oficial era contundente:

Toca pues al gobierno y a los hombres ilustrados y de elevados y cristianos sentimientos impulsar por cuantos medios sea posible la educación popular base natural del progreso y la libertad, luchando para ello sin tregua alguna contra la indiferencia general de los padres de familia y contra los peores egoístas del mundo, como son los anti-instruccionistas. (Echeverri-Álvarez, 2015)

$Y$ si no eran suficientes las "amonestaciones hechas a los padres, guardadores, maestros 0 patronos" y se infringían las disposiciones de enviar los niños a las escuelas, se emplearían medidas contundentes: "los niños serán conducidos a las escuelas por un agente de policía, i los que tengan a su cargo serán gradualmente conminados con multa de veinte pesos 0 arresto hasta de 10 días" (Echeverri-Alvarez, 2015). Como se ve, la fuerza fue parte de las estrategias para alentar la relación entre escuela y familia, esfuerzo en el que todavía se asume por otras vías. Ciertamente se han ido tejiendo, paulatinamente, formas cada vez más exhaustivas de esa relación, aunque siempre se mantengan, para poder sostenerla, las tensiones, las denuncias reciproca, los esfuerzos, los llamados a mejores acercamientos y las distancias prudentes. Múltiples serían los ejemplos de esa tensión durante los siglos XX y XXI. Baste un ejemplo de esta complicada relación histórica entre escuela y familia de finales del siglo pasado.

Las investigadoras Ceballos y Galeano (2014), reconocían, como maestras de escuela, que estas relaciones son complejas, forzadas de alguna manera, interesadas, en fin, las llaman de manera abarcativa como "relaciones paliativas": puesto que mitigan, atenúan o suavizan los efectos adversos que se producen en las dinámicas cotidianas de la escuela, más que ser un asunto de concertación, diálogo y construcción de la misma escuela.

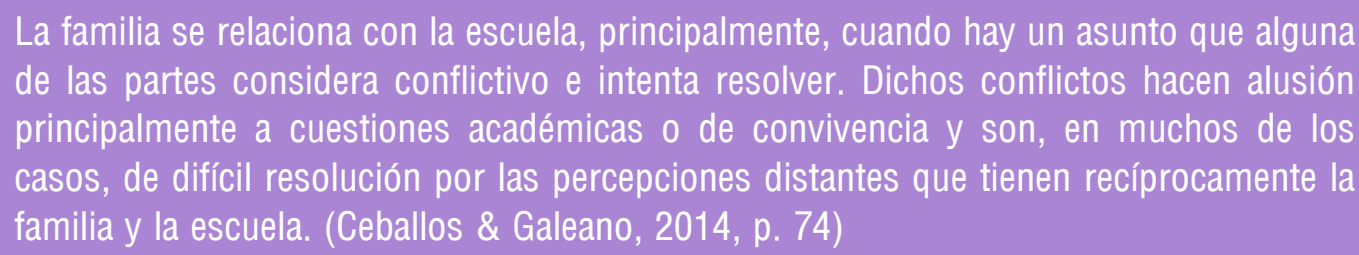

En fin, cuando se habla de "educación afectivo-sexual en las escuelas familiares", no se juntan elementos conflictivos por inocencia, ni se invoca sin más ideales de teleologías educativas, sino que se trabaja con base en el presupuesto de que es posible darle otra vuelta de tuerca a las relaciones familia escuela para abarcar cada vez más dimensiones de lo humano para el bienestar de las personas, el mejoramiento del tejido social y el fortalecimiento de la democracia. Por tanto, escuelas familiares para la educación afectivo-sexual, es una apuesta que no castiga, impone 
multas 0 juzga desde las orillas de la escuela o de la familia la contraparte de la corriente, sino que se esfuerzan en mejorar el cauce de la educación integral para el bienestar físico y mental de las personas.

El ejercicio de reflexión pensado para acompañar a los sujetos en su autonomía es de vital importancia en la medida en que participa tanto la familia como la escuela. Estos dos ámbitos de vida son indispensables para la construcción afectivo-sexual, debido a la influencia en la práctica cotidiana de vida, que pasa por el ser y se visibiliza en el hacer social característico de la personalidad.

Así las cosas, la sexualidad humana es el resultado de la interacción cognitiva y afectiva de los individuos, su medio o cultura, respecto a las posibilidades de expresión de comportamientos sexuales, afectivos, placenteros y filogenéticos (Barragán, 1991). Se practica de manera interaccional con otros, no obstante, no se prepara ni en la escuela o la familia para la misma, es decir, persiste la necesidad de reflejar de una educación sexual plena en la interacción social. Sólo cuando se hace evidente la expresión de la sexualidad como acción de (auto)reconocimiento del cuerpo, las emociones, la palabra y el gusto hacia otros, es que aparece la familia y la escuela a intervenir en la formación de lo que se considera una educación sexual plena y activa. Ambas por separadas, y pocas veces se unen para tal fin.

Por un lado, la participación de la familia es importante en la medida en que dentro de las dinámicas internas se posibilita el diálogo como formador de identidades de manera respetuosa. Estos espacios toman una coloración educativa e interactiva frente a lo que se ha vivido y conocido al respecto, es decir, cada integrante pone sobre la mesa el significado y sentido que le dan a la sexualidad y afectividad, y mediante este se generan, con la alteridad siempre presente, unos aprendizajes a la luz de la historia, la cultura y las permanencias sociales.

De esta manera, la familia se convierte en el escenario que brinda la pauta para el ejercicio colaborativo con los niños, niñas y adolescentes, pues ellos, son quienes preguntan desde una postura del "no saber". Hernández-Prados et al. (2018) hacen un llamado de atención a los niveles de participación que presentan las familias en los procesos educativos con los hijos.

Al interior de la familia existe la posibilidad de visibilizar un trazo subjetivo y sensible a lo que la educación sexual respecta, es necesaria la articulación de otros ámbitos transversales de vida por los que pasan los seres humanos y dejan huella en la construcción de identidad, personalidad y ciudadanía, la escuela, respectivamente. Por otro lado, la participación de la escuela en este tema converge y diverge, en ocasiones, lo aprehendido en la familia; inicialmente, se unen en la intención de aportar a la autonomía que debe tener cada persona para ejercer el derecho libre y responsable de lo que respecta a la sexualidad y la afectividad; no obstante, divergen en la forma en que generan espacios para el diálogo reflexivo, crítico, educativo, también, en el devenir subjetivo que trae consigo tratar un tema que para algunos maestros y maestras es considerado un tabú y desde este paradigma es que se comparten los espacios de aprendizaje. 
Antes de que la institucionalidad tome cartas en el asunto, es indispensable, en ocasiones, generar un trabajo previo con las familias, maestros y maestras para desaprender lo aprendido de la sexualidad y la afectividad, ya que se generan con los alumnos diálogos pedagógicos que ponen en tela de juicio experiencias culturales, históricas y subjetivas. Barrientos et al. (2018) refieren que "las escuelas continúan reproduciendo estereotipos y visiones rígidas sobre el género y la sexualidad, las cuales generan desigualdad, exclusión y violencia, problemáticas que a menudo son invisibles para las comunidades escolares" (p. 2).

Generalmente, la intersección que existe entre la familia y la escuela para poder aportar a la construcción social y humana de los sujetos se encuentra en las escuelas familiares; lugar donde se unen los actores, diálogos y prácticas para que los niños, niñas y adolescentes se permitan crecer con una autonomía, libertad y formas de expresar corporalmente sus emociones. El lenguaje del cuerpo manifiesta emociones inconscientes a otra persona que llama la atención para construir proyectos de vida; no obstante, mal trabajada la educación sexual, este tema es engorroso tanto para las familias, el profesorado como para el alumnado.

Las escuelas familiares son estrategias institucionalizadas para promover la participación de la familia en la escuela y desde allí se generen acciones de bienestar para los niños, niñas y adolescentes en perspectiva de género (Valdivielso et al., 2016). Dentro de las acciones permeadas debe tratarse de fomentar la capacidad de agencia como alternativa de vida para una educación afectivo-sexual plena.

\section{Las escuelas familiares: territorio de encuentro que agencia la educación afectivo-sexual}

Las escuelas familiares bajo la denominación de escuelas de padres² ${ }^{2}$ se convierten en el lugar que vincula a las familias con las instituciones educativas; no obstante, ese vínculo se ha construido desde la información y no desde la formación; los padres asisten para conocer las notas de sus hijos, lo que han hecho bien o mal en el período académico y no se genera un proceso reflexivo con los maestros para articular acciones en pro de los niños, niñas y adolescentes. Por lo que el reto consiste en realizar una modificación en los discursos y las prácticas para la comprensión de este escenario. En una investigación realizada en Colombia, las escuelas familiares son reconocidas como una estrategia socioeducativa (Rodríguez Triana \& Suárez Ortiz, 2019), que favorece los procesos de desarrollo humano y social de cada persona que interactúa en la relación familia y escuela. La categoría se asume en el devenir del texto como el 
Asumir las escuelas familiares como territorio de encuentro reconoce a las familias y a las escuelas como entornos educativos que favorecen la constitución de lo humano a favor de los $N_{N A}{ }^{3}$ quienes en el cruce de la relación requieren que sus dos más cercanos educadores se comprometan en un dialogo permanente. Así, la comprensión de la escuela familiar como territorio educativo la sitúa como una (Rodríguez Triana \& Suárez Ortiz, 2019) comunidad de prácticas en la que además de crear lazos de interacción y reconocimiento, se compartan conocimientos, intenciones y códigos simbólicos para aportar a la formación de la autonomía, la autorregulación, la otredad y la sensibilidad de los sujetos que tienen en medio.

Las escuelas familiares, según las comprensiones del profesor Calvo (2008), son un territorio de encuentro que agencia la educación afectivo-sexual por cuanto, desde lo cultural la relación entre familia y escuela, ambos actores, a través del proceso educativo compartido, se comprometen en la comprensión y educación de la sexualidad más allá de condiciones biológicamente instaladas para asumirla desde lo cultural y lo diverso. El territorio educativo, desde la escuela familiar, traspasa las fronteras de la escuela para hacer posible la creación de relaciones posibles donde se haga presente la individuación y la relación; al tiempo de la creación, el goce por encima de la repetición, la negación y la exclusión.

Las escuelas familiares como territorio de encuentro es una estrategia pedagógica, que incluida y formalizada en los proyectos educativos institucionales sitúa a la familia, en los procesos escolares y educativos con todo lo que ella es y representa, y significa su lugar y participación para construir códigos compartidos que le permitan a los NNA la propensión para aprender y no para repetir y que encuentren en los contenidos propios de lo afectivo-sexual voces en coherencia, en un tema sustantivo para sus vidas. En esta línea, se reconocen los espacios que genera el diálogo reflexivo y autónomo para la construcción de identidad individual y social; logran tener diferentes denominaciones, trabajos previos para la comprensión de su significado, y por eso, aparece la categoría de Educación Sexual Integral (ESI) en perspectiva de género, la cual es considerada

dentro del campo educativo, [...] [la que] potencia prácticas pedagógicas críticas y puede ser un punto de partida para reformular las relaciones sociales de géneros heteronormativas y patriarcales, para hacer más digna la vida humana entendiendo que asumimos la necesidad de modificar otros patrones de dominación capitalista y colonial. (Talani Zuvela, 2019, p. 13)

Así las cosas, el reconocimiento de la categoría género en su máxima expresión alude también a la generación de espacios reflexivos en las escuelas familiares, pues la permanencia y confianza brindada por las familias a la institución reflejan compromiso y reflexión profunda en el tema. Si bien el ejercicio formativo de los seres humanos es una tarea tanto de la familia como de la escuela, pareciera ser que, en ocasiones, cada una va por un camino diferente de motivaciones y 
reflexiones. Por eso, "se hace necesario un acercamiento en el que la educación familiar se coloque como posibilidad pedagógica de tal confluencia" (Páez \& Pérez, 2018, p. 9). En este llamado, es que las escuelas familiares se convertirán en un lugar que teja y una educativamente temáticas relacionadas con lo afectivo-sexual. Los maestros y maestras son quienes deben resignificar las concepciones que tienen frente a la temática para poder promover la reflexión en el aula. Barrientos et al. (2018) refieren que "adquirir conceptos propios de los estudios de género debe permitir que los docentes sean capaces de reflexionar y abrir discusiones con perspectiva de género sobre la escuela" (p. 4). Por lo cual, la clave se encuentra en la intención de repensar lo aprehendido para fomentar un aprendizaje colectivo.

Por eso, es que el ejercicio reflexivo y de resignificación conceptual frente a la afectividad y sexualidad se convierte en una construcción vital para el ser humano en lo subjetivo e interpretativo de la realidad. La familia aporta desde su experiencia de vida y la institución amplía la mirada para el acompañamiento integral de los niños, niñas y adolescentes. Pensar en la educación afectivo sexual en las escuelas familiares, supone hacer una revisión a las teorías feministas para identificar que la violencia ejercida desde el sistema patriarcal se debe a una necesidad significativa en el ejercicio educativo como ciudadanos, una deuda que desde lo político y las organizaciones continúa siendo un pendiente por resolver. (Barragán Gamba, 2015, p. 205)

A manera de conclusión, los sujetos están en constante movimiento entre la familia y la escuela que dinamizan territorios convergentes y divergentes en cuanto a la educación afectivo-sexual. Esta temática señalada genera discusiones desde lo construido por cada actor, la familia y la escuela; para ello, se propone un lugar común que motiva la resignificación y reflexión de lo sexual y afectivo entre los seres humanos, las prácticas, concepciones y lugares ocupados. Las escuelas familiares permiten dinamizar interacciones en pro de los niños, niñas y adolescentes que dan respuesta a sus necesidades en los procesos de cuidado, crianza y socialización constante, en el escrito, es clara la propuesta realizada para pensar la educación afectivo-sexual sin descuidar la implicación que genera la connotación de género en lo subjetivo y la manera en que los maestros permiten ser y hacer distinto en la actualidad. La promoción de estrategias pedagógicas que se ajusten a las necesidades de las familias en las escuelas familiares muestra un avance para la comprensión y construcción vital.

La familia y la escuela están invitadas a trabajar en conjunto para el desarrollo humano de los sujetos y en su máxima expresión se piensan las escuelas familiares como territorio que unifica y muestra el aporte que realiza uno u otro en el sujeto. Esta tarea debe responderse de manera formativa y no informativa. Pues la segunda limita las capacidades humanas en materia de libertad, autonomía y gestión subjetiva.

La invitación es para las disciplinas que reflexionan a la luz de las instituciones educativas, maestros, familias y el alumnado, en el trabajo articulado, reconociendo cada actor desde su diferencia y potencial para el cambio. Pensar la educación afectivo-sexual en la actualidad se plantea con retos y fronteras por deconstruir, motivo por el cual se invita a que se continúe con el aporte a la temática como formación integral de los seres humanos. Buscar alternativas para 
que las temáticas propuestas en este documento no se aborden desde los prejuicios morales de quienes fomentan los espacios, sino que se busque la manera de educar en libertad, de forma crítica y reflexiva en beneficio de la sociedad.

En suma, las escuelas familiares son territorios de encuentro donde las familias y las instituciones educativas, como agentes primarios en la formación humana, interactúan para vivenciar un proceso que les permita crear relaciones posibles situadas en sus experiencias y sentimientos, así también en conocimientos elaborados; todo ello unido, permite respuestas explicativas y comprensivas al mundo que les rodea en virtud de sus NNA, y en este caso en particular en lo referido a la educación afectivo-sexual en la que se signifique el reconocimiento propio y del otro y se asuma el cuerpo y la corporeidad desde el goce único y sinérgico. De esta manera, una escuela familiar en esta comprensión y en este tema en especial, aviva en las familias y maestros el interés por aprender y permitirle a sus NNA la curiosidad, la exploración y la pregunta sobre la sexualidad como un componente eminentemente humano.

\section{Consideraciones finales sobre el ejercicio de la pasantía como intercambio de saberes y experiencias de acuerdo con el reglamento estudiantil de formación avanzada de la Universidad Pontificia Bolivariana}

ARTICULO $34^{\circ}$. La pasantía de investigación es una estancia investigativa de carácter nacional 0 internacional, que se realiza en grupos de alto nivel de conocimiento, centros, institutos, facultades o programas de impacto, que aporta al desarrollo del trabajo de grado o de la tesis e incluye actividades académicas directamente relacionadas con el trabajo de investigación que contribuyan a consolidar las competencias en investigación.

ARTíCULO $35^{\circ}$. Pasantía académica es la estancia encaminada a reforzar la formación del estudiante mediante la interacción intercultural, la capacitación complementaria en otras metodologías de trabajo científico, la consulta de fondos bibliográficos o documentales, el aprendizaje de técnicas instrumentales o metodológicas, la asistencia a cursos especializados relacionados con su área de formación y la participación en programas académicos de otras instituciones que ofrezcan seminarios avanzados o la realización de un ejercicio aplicado.

ARTíCULO $36^{\circ}$. La duración máxima y el número de pasantías académicas o de investigación será determinada por los requerimientos del programa 0 del proyecto de investigación y las entidades 0 instituciones involucradas en su desarrollo, previa aprobación del coordinador del programa, y en el caso de doctorados, del Comité Tutorial. En todos los casos el director del proyecto y el coordinador del programa acordarán con el estudiante los mecanismos de contacto que permitan hacer un seguimiento. La matrícula de estos créditos debe ser realizada en los tiempos establecidos por la Universidad para la matrícula. Parágrafo $1^{\circ}$. A 
cada pasantía se le asignará un valor en créditos correspondiente a alguna de las actividades académicas específicas del programa. En ningún caso los créditos de pasantía sumados a las demás actividades académicas deben exceder el total de créditos del programa. Parágrafo $2^{\circ}$. La gestión y consecución de los recursos para las pasantías investigativa 0 académica estarán a cargo del estudiante.

ARTÍCULO $37^{\circ}$. Al finalizar cada pasantía, el estudiante presentará un informe de las actividades realizadas y de los logros alcanzados, al coordinador del programa 0 , en caso de los doctorados al Comité Tutorial. El director del proyecto 0 el docente responsable del seguimiento de las actividades de la pasantía emitirá una nota cualitativa, de acuerdo con el desempeño del estudiante. Los cursos realizados en el marco de las pasantías académicas podrán ser sometidos a homologación. (Universidad Pontificia Bolivariana, 2021)

\section{Agradecimientos}

Al Doctor Joaquín Parra Martínez, contacto directo por parte de la Universidad de Murcia (España); al Departamento de Métodos de Investigación y Diagnóstico en Educación; Facultad de Educación y al Grupo Investigación "Compartimos Educación" del mismo claustro, por su acogida y permanencia durante el proceso de pasantía internacional entre la Universidad Pontificia Bolivariana (Medellín) y su Doctorado en Educación, posgrado que hace parte de los Programas de la Escuela de Educación y Pedagogía de la UPB.

\section{Conflicto de intereses}

Los autores declaran la inexistencia de conflicto de interés con institución o asociación comercial de cualquier índole.

\section{Referencias}

Barragán-Gamba, D. (2015). La violencia contra las mujeres es cuestión de género. Criterio Libre Jurídico, 12(2), 79-90. https://revistas.unilibre.edu.co/index.php/criteriojuridico/article/ view/574/441 
Barragán, M. F. (1991). "Programa de educación afectivo sexual. Educación secundaria” I. Sexualidad, educación sexual y género. Junta de Andalucía. Consejería de Educación y Ciencia e Instituto Andaluz de la Mujer.

Barrientos, P., Andrade, D., \& Montenegro, C. (2018). La formación docente en género y diversidad sexual: tareas pendientes. Cuaderno de Educación, (81), 1-13. https://www.academia. edu/38576396/La_formaci\%C3\%B3n_docente_en_g\%C3\%A9nero_y_diversidad_sexual_ Tareas_pendientesBejarano Franco, M. T., \& García Fernández, F. B. (2016). La educación afectivo-sexual en España. Análisis de las leyes educativas en el periodo 1990-2016. Opción, 32(13), 756-789. https://www.produccioncientificaluz.org/index.php/opcion/article/ view/21624/21427

Bejarano Franco, M., \& Mateos Jiménez, A. (2015). La educación afectivo-sexual en el sistema educativo español: análisis normativo y posibilidades de investigación. Revista Ibero-Americana de Estudos em Educação, 10(2), 1507-1522. https://doi.org/10.21723/riaee.v10i6.8334

Carmona Sáez, P., Parra Martínez, J., \& Gomariz Vicente, M. A. (2021). Participación de las familias de alumnado con apoyos y atenciones diferentes: un estudio en un contexto multicultural. Revista de Investigación Educativa, 39(1), 49-69. https://doi.org/10.6018/rie.386551

Ceballos, A., \& Galeano, E. (2014). La relación familia-escuela: una pregunta desde la convivencia y el desempeño académico de los estudiantes de básica primaria de dos instituciones educativas del área metropolitana [Tesis de Maestría, Universidad Pontificia Bolivariana].

Del Pozo, F., Aparico, J. A., \& Rodríguez, Z. (2020). Educación familiar y escolar en Colombia: Cruces de caminos para la construcción de paz comunitaria. En M. Saracostti \& L. Lara. Avanzando hacia relaciones más colaborataivas entre familias y escuela: Experiencias desde países iberoamericanos. Universidad Autónoma de Chile. https://bit.ly/3C3J55I

Echeverri-Álvarez, J. (2015). Escuela y métodos pedagógicos en clave de gubernamentalidad liberal: Colombia, 1821-1946. Universidad Pontificia Bolivariana.

Font, P. (2010). Educación afectiva y sexual en la escuela. Orientaciones para maestros y maestras. Instituto de Estudios de la Sexualidad y la Pareja.

Garín, D. F., Meza, L. Y., \& Plaza, D. A. (2016). Formación en derechos sexuales y reproductivos con enfoque de género [Tesis inédita de pregrado, Universidad Academia de Humanismo Cristiano].

Gomariz Vicente, M. A., Parra Martínez, J., García Sanz, M. P., \& Hernández Prados, M. A. (2019). De lo formal a lo real. Análisis de la participación familiar en asociaciones de madres y padres y consejos escolares. Aula Abierta, 48(1), 85-96. https://doi.org/10.17811/rifie.48.1.2019.85-96

González Gómez, Y. (2015). El papel del docente en la educación para la sexualidad: algunas reflexiones en el proceso educativo escolar. Actualidades Investigativas en Educación, 15(3), 1-15. http://dx.doi.org/10.15517/aie.v15i3.20335 
Hernández-Prados, M. A., García-Sanz, M.-P., Parra-Martínez, J., \& Gomariz Vicente, M. A. (2018). Participación familiar en los centros de educación secundaria. Anales de Psicología, 35(1), 84-94. https://doi.org/10.6018/analesps.35.1.325981

Lamas, M. (1996). El género. La construcción cultural de la diferencia sexual. PUEG.

Langa Herrero, A. (2019). La incorporación de los derechos humanos a las metodologías de cooperación al desarrollo a través del enfoque de derechos y la acción sin daño. Revista Internacional de Pensamiento Político, (14), 251-267. https://doi.org/10.46661/revintpensampolit.4783

Lazo Corvera, P., \& Collignon Goribar, N. M. (2017). Derechos humanos/sexuales, género y biopolítica: reflexiones sobre la configuración subjetiva del derecho a la libre elección de identidad de género. La ventana. Revista de Estudios de Género, 5(45), 50-87. https://doi.org/10.32870/ Iv.v5i45.5349

Congreso de Colombia. (2006). Ley 1098 de 2006. https://www.icbf.gov.co/cargues/avance/docs/ ley_1098_2006.htm

López, B. M. I. (2016). Actitudes y creencias de padres y madres hacia la educación sexual [Tesis inédita de maestría, Universidad Jaume l].

Organización Mundial de la Salud. (2006). Colaboremos por la salud. Informe de la salud en el mundo. https://apps.who.int/iris/handle/10665/43434

Páez, R. M., \& Pérez, N. (2018). Educación familiar. Investigación en contextos escolares. CLACSO.

Rodríguez Bustamante, A., \& Viveros Chavarría, E. F. (2013). Sobre la intervención familiar en escenarios educativos. Aproximación a una revisión documental. Katharsis, (15), 113-132. https://revistas.iue.edu.co/index.php/katharsis/article/view/241/393

Rodríguez Bustamante, A., Agudelo Gallego, C., \& Córdoba-Quintero, L. (2021). Lo sistémico entre escuela y familia. Universos posibles. Perseitas, 9. https://doi.org/10.21501/23461780.3975

Rodríguez, Triana, Z. E., \& Suárez Ortiz, J. L. (2019). Escuelas familiares. Una experiencia favorecedora para el desarrollo humano. Revista Electrónica Interuniversitaria de Formación del Profesorado, 22(3), 127-138. https://doi.org/10.6018/reifop.390161

Rodríguez Triana, Z. E. (2014). Escuelas familiares. Escenarios para el encuentro. Departamento de Estudios de Familia. Universidad de Caldas.

Rodríguez Triana, Z. E. (2018). Qué y cómo se enseña y aprende en la familia. Un asunto de interés para la escuela. Revista Latinoamericana de Estudios Educativos, 14(2), 132-157. http://latinoamericana.ucaldas.edu.co/downloads/Latinoamericana14(2)_7.pdf

Rosales, M. A. L., \& Flores, S. A. (2009). Género y sexualidad en las universidades públicas mexicanas. Iconos. Revista de Ciencias Sociales, (35), 67-75. https://revistas.flacsoandes.edu. ec/iconos/article/view/377Talani Zuvela, P. (2019). Educación sexual integral y perspectiva de 
género en la formación docente: una conjunción indispensable para una pedagogía crítica y feminista. Saberes y prácticas. Revista de Filosofía y Educación, 4, 1-16. https://revistas.uncu. edu.ar/ojs/index.php/saberesypracticas/article/view/2197

Universidad Pontificia Bolivariana (2021). Reglamento Estudiantil de Formación Avanzada. Consejo Directivo General Acuerdo CDG No. 24/2014 (25 de septiembre de 2014). https://gconocimiento.upb.edu.co/gesdoc/Informacin\%20Institucional/Reglamento\%20Estudiantil\%20de\%20 Formaci\%C3\%B3n\%20Avanzada\%202014.pdf

Valdivielso, S., Ayuste, A., Rodríguez, M., C., \& Vila, E., S. (2016). Educación y género en la formación docente en un enfoque de equidad y democracia. En Carrillo, I. (Coord.), Democracia y educación en la formación docente, (pp. 117-140). Universitat de VicUniversitat Central de Catalunya. 